\title{
PRÁTICAS OBSTÉTRICAS DE UMA MATERNIDADE PÚBLICA EM RIO BRANCO- $\mathrm{AC}^{*}$
}

\author{
Sheley Borges Gadelha de Lima ${ }^{1}$, Janine Schirmer ${ }^{2}$, Leila Maria Geromel Dotto ${ }^{3}$, Clisângela Lago Santos ${ }^{4}$
}

\begin{abstract}
RESUMO: Objetivo: identificar as práticas obstétricas na assistência ao parto e nascimento em uma maternidade pública de Rio Branco, Acre. Método: estudo descritivo exploratório de delineamento transversal, com 460 puérperas de partos vaginais e de seus recém-nascidos ocorrido de novembro de 2013 a fevereiro de 2014. Dados coletados por meio de entrevistas, prontuário e folha de admissão do recém-nascido, submetidos à análise estatística descritiva e analítica. Resultados: práticas demonstradamente úteis: Presença de acompanhante $400(87 \%)$; oferecimento de dieta 349 (75,9\%); liberdade de posição e movimento 374 (81,3); uso de partograma $246(53,5 \%)$; alívio da dor 360 (78,3\%) e contato pele a pele $433(94,1 \%)$. Práticas inapropriadas: Amniotomia 249 (54,2\%). Práticas claramente prejudiciais: infusão de ocitocina 87 (39\%), posição horizontal para o parto 435 (94,6\%), manobra de Kristeller no período expulsivo 71 (15,5\%). Conclusão: é possível romper com o modelo obstétrico praticado atualmente, porém incentivos na formação dos profissionais se fazem necessários.

DESCRITORES: Parto humanizado; Enfermagem obstétrica; Trabalho de parto; Humanização da assistência.
\end{abstract}

\section{OBSTETRIC PRACTICES ADOPTED BY A PUBLIC MATERNITY IN RIO BRANCO-AC}

ABSTRACT: Objective: To identify the obstetric practices used in childbirth and birth care in a public maternity hospital in Rio Branco, Acre. Method:Descriptive exploratory cross-sectional study with 460 women who had vaginal deliveries and their newborns from November 2013 to February 2014. Data was collected through interviews, medical records and documentation related to the newborn and submitted to descriptive and statistical analysis.Results:Demonstrably useful practices: Presence of a companion 400 $(87 \%)$; use of a light diet 349 (75.9\%); freedom of position and movement 374 (81.3); use of partograph forms in 246 (53.5\%); pain relief $360(78.3 \%)$ and skin to skin contact $433(94.1 \%)$. Inappropriate practices: Amniotomy $249(54.2 \%)$. Clearly harmful practices: oxytocin infusion 87 (39\%), horizontal position during fetal expulsion 435 (94.6\%), Kristeller maneuver in the expulsionperiod 71 (15.5\%). Conclusion:The obstetrical model currently used can be abandoned, but this involves support to the training of health professionals. DESCRIPTORS: Humanized birth; Obstetric nursing; Labor; Humanization of care.

\section{PRÁCTICAS OBSTÉTRICAS DE UNA MATERNIDAD PÚBLICA EN RIO BRANCO-AC}

RESUMEN: Objetivo: Identificar las prácticas obstétricas en la atención del parto y nacimiento en una maternidad pública de Rio Branco, Acre. Método: Estudio descriptivo exploratorio de delineamiento transversal, con 460 puérperas de partos vaginales y de sus recién nacidos, realizado entre noviembre de 2013 y febrero de 2014. Datos recolectados mediante entrevistas, historia clínica y hoja de admisión del recién nacido, sometidos a análisis estadístico descriptivo y analítico. Resultados: Prácticas decididamente útiles: Presencia de acompañante 400 (87\%); ofrecimiento de dieta 349 (75,9\%); libertad de posición y movimiento 374 (81,3\%); uso de partograma 246 (53,5\%); alivio del dolor 360 (78,3\%) y contacto piel a piel 433 (94,1\%). Prácticas inadecuadas: Amniotomía 249 (54,2\%). Prácticas decididamente perjudiciales: infusión de oxitocina 87 (39\%); posición horizontal para el parto 435 (94,6\%); maniobra de Kristeller en fase de expulsión 71 (15,5\%). Conclusión: Es posible abandonar el modelo obstétrico actual, resultando necesarios incentivos en la formación de profesionales.

DESCRIPTORES: Parto Humanizado; Enfermería Obstétrica; Trabajo de Parto; Humanización de la Atención.

\footnotetext{
*Artigo extraído da dissertação de mestrado: "Atenção ao trabalho de parto e parto em uma Maternidade do SUS, na capital do Acre". Universidade Federal de São Paulo, 2014.

${ }^{1}$ Enfermeira. Mestra em Ciências. Docente da Universidade Federal do Acre. Rio Branco, AC, Brasil.

${ }^{2}$ Enfermeira. Doutora em Enfermagem. Docente de Enfermagem da Universidade Federal de São Paulo. São Paulo, SP, Brasil.

${ }^{3}$ Enfermeira. Doutora em Enfermagem. Docente da Universidade Federal do Acre. Rio Branco, AC, Brasil.

${ }^{4}$ Enfermeira. Doutora em Ciências. Docente da Universidade Federal do Acre. Rio Branco, AC, Brasil.
}

Autor Correspondente:

Sheley Borges Gadelha de Lima

Universidade Federal do Acre

R. Jatobá, 70 - 69921-118 - Rio Branco, AC, Brazil

Email: sheley.lima@gmail.com
Recebido: $14 / 06 / 2017$

Finalizado: 03/10/2018 


\section{INTRODUÇÃO}

Ao longo do tempo instaurou-se a ideia de que o nascimento só seria seguro quando acompanhando por um profissional médico, tornando-o a figura central deste processo, voltando a atenção mais para os processos fisiológicos do que para as necessidades das mães e de seus filhos ${ }^{(1)}$.

Segundo a Organização Mundial de Saúde (OMS), há evidências científicas de que várias práticas assistenciais, durante a gestação e parto, são promotoras de melhores resultados obstétricos, com impacto positivo nos desfechos perinatais. Grande parte das complicações ocorridas durante o processo do trabalho de parto e parto podem ser evitadas com a assistência obstétrica apropriada e o uso pertinente de tecnologia ${ }^{(2)}$. Vale ressaltar que o uso impróprio de tecnologias e intervenções pode acarretar um desfecho adverso tanto para a mãe quanto para seu concepto ${ }^{(2-4)}$.

Na tentativa de melhorar a assistência obstétrica, foram sendo criadas leis, programas, incentivos oficiais, em busca de assistência humanizada e segura. A estratégia mais recente foi lançada em 2011, pelo Ministério da Saúde (MS), sendo chamada de Programa Rede Cegonha ${ }^{(5)}$.

Diante disso, torna-se importante identificar como estão sendo realizadas as práticas obstétricas de uma maternidade pública da capital do Estado do Acre e se estão sendo realizadas em consonância com o recomendado pela OMS e MS.

\section{- MÉTODO}

Trata-se de estudo descritivo e exploratório de delineamento transversal sobre a assistência obstétrica oferecida na atenção ao trabalho de parto (TP) e parto em uma maternidade do SUS, no município de Rio Branco, Estado do Acre, no período de novembro de 2013 a fevereiro de 2014.

A pesquisa incluiu todas as mulheres que tiveram parto vaginal, com conceptos vivos ou mortos, com peso ao nascer $\geq 500 \mathrm{~g}$ e/ou idade gestacional $\geq 22$ semanas de gestação, destas 460 mulheres aceitaram participar do estudo. Foram excluídas as puérperas que não entendiam a língua portuguesa, com necessidades especiais ou deficientes (que tinham incapacidade de responder a entrevista).

O instrumento de pesquisa foi preenchido através de entrevista estruturada, dados do cartão pré-natal, do prontuário da puérpera e da folha de admissão do recém-nascido. Este foi adaptado da pesquisa "Saúde Reprodutiva de Primigestas: Análise de fatores relacionados ao tipo de parto" ${ }^{(6)}$.

Foram analisadas as práticas de acordo com a categorização lançada pelo MS em 2001, que teve como base a classificação da OMS lançada em $1996^{(7)}$.

As variáveis qualitativas foram descritas com número e porcentagem; as variáveis quantitativas, como média \pm desvio padrão para as variáveis que se mostraram normalmente distribuídas ou, como mediana ( $1^{\circ}$ quartil - $3^{\circ}$ quartil), valores de mínimo e máximo no caso da distribuição Gaussiana não se ajustar aos dados.

Os dados foram digitados em planilhas do Excel 2010. Para a análise estatística utilizou-se o programa SPSS 16.0 e para a generalização do Teste Exato de Fisher utilizou-se o programa STATA/SE 13.0.

Neste estudo foram observados os princípios éticos da Resolução $n^{\circ} 466 / 12$, do Conselho Nacional de Saúde, possuindo aprovação no Comitê de Ética em Pesquisa no dia 05 de julho de 2013, sob parecer $n^{\circ} 319.809$.

\section{- RESULTADOS}

Foram observadas as práticas recomendadas pela OMS e MS, entre as demonstradamente úteis que devem ser estimuladas foram: a ingesta de líquidos durante o trabalho de parto, enquanto estavam internadas na sala de pré-parto, parto e pós-parto (PPP), referida por 349 (75,9\%). Quanto à presença do acompanhante, 400 (87\%) mulheres responderam que ficaram com acompanhante durante seu 
período de internação na maternidade.

No tocante à movimentação, 201(43,7\%) responderam que ficaram a maior parte do tempo no leito, em contrapartida $374(81,3 \%)$ das participantes disseram terem sido orientadas/estimuladas a levantar e mudar de posição, 360 (78,3\%) foram orientadas a fazer exercícios, deambular foi o mais aderido, $317(88,1 \%), 245(68,1 \%)$ realizaram exercício na bola e banho foi realizado por $227(63,1 \%)$ gestantes, o exercício menos realizado foi com o cavalinho 102 (28,3\%), sendo que 231 (64,3\%) receberam massagem de conforto durante o trabalho de prato.

A administração de ocitócitos no terceiro período do trabalho de parto foi realizada em 421 (90\%) das mulheres entrevistadas (Tabela 1).

Tabela 1 - Distribuição numérica e proporcional, práticas no parto normal consideradas úteis e que devem ser estimuladas, segundo OMS. Rio Branco, AC, Brasil, 2014

\begin{tabular}{|c|c|c|c|}
\hline \multicolumn{2}{|c|}{ Práticas no parto normal consideradas úteis e que devem ser estimuladas } & \multirow{2}{*}{$\frac{\mathbf{N}}{349}$} & \multirow{2}{*}{$\begin{array}{c}\% \\
75,9\end{array}$} \\
\hline Durante o trabalho de parto no & Sim & & \\
\hline hospital, pode ingerir líquidos & Não & 104 & 22,6 \\
\hline & Não informado & 7 & 1,5 \\
\hline \multirow{4}{*}{$\begin{array}{l}\text { Teve acompanhante durante } \mathrm{o} \\
\text { trabalho de parto e parto }\end{array}$} & Sim, em ambos TP e Parto & 400 & 87 \\
\hline & Sim, só no TP & 4 & 0,9 \\
\hline & Não, porque não sabia que podia ou porque não queria & 19 & 4,1 \\
\hline & Não, porque não tinha ninguém para ficar & 37 & 8 \\
\hline \multirow{2}{*}{$\begin{array}{l}\text { A pessoa que ficou no trabalho e } \\
\text { parto era de sua escolha (n 404) }\end{array}$} & Sim & 345 & 85,4 \\
\hline & Não & 59 & 14,6 \\
\hline \multirow{2}{*}{$\begin{array}{l}\text { Durante o TP permaneceu a } \\
\text { maior parte do tempo no leito }\end{array}$} & Sim & 201 & 43,7 \\
\hline & Não & 259 & 56,3 \\
\hline \multirow{2}{*}{$\begin{array}{l}\text { Durante o TP foi estimulada a } \\
\text { caminhar e mudar de posição }\end{array}$} & Sim & 374 & 81,3 \\
\hline & Não & 86 & 18,7 \\
\hline \multirow{2}{*}{$\begin{array}{l}\text { Durante o trabalho de parto foi } \\
\text { orientada a fazer exercícios }\end{array}$} & Sim & 360 & 78,3 \\
\hline & Não & 100 & 21,7 \\
\hline \multirow[t]{5}{*}{ Exercícios orientados } & Caminhar & 317 & 88,1 \\
\hline & Bola & 245 & 68,1 \\
\hline & Massagem de conforto & 231 & 64,2 \\
\hline & Banho & 227 & 63,1 \\
\hline & Cavalinho & 102 & 28,3 \\
\hline \multirow[t]{2}{*}{ Partograma preenchido } & Sim & 246 & 53,5 \\
\hline & Não & 214 & 46,5 \\
\hline \multirow{2}{*}{$\begin{array}{l}\text { Partograma registrado de forma } \\
\text { completa ( } \mathrm{n} 246)\end{array}$} & Sim & 105 & 42,7 \\
\hline & Parcialmente & 141 & 57,3 \\
\hline \multirow{3}{*}{$\begin{array}{l}\text { Uso de ocitocina após expulsão } \\
\text { da placenta }\end{array}$} & Sim & 421 & 91,7 \\
\hline & Não & 39 & 8,3 \\
\hline & Não informado & 12 & 2,6 \\
\hline
\end{tabular}

A totalidade das mulheres, ou seja, 460 não realizaram enema em preparação ao parto, sendo esta uma prática abolida desta instituição. A tricotomia pubiana apareceu em 415 (90,4\%) das parturientes, porém, a grande maioria 405 (88\%) realizou em seu domicílio; $223(48,5 \%)$ receberam infusão venosa, destas 87 (39\%) não sabiam se tinha medicamento no soro, e grande parte 139 (62,3\%) desconheciam o motivo de estarem recebendo infusão venosa. 
Quanto às posições no período expulsivo, foram mais adotadas as deitada e semi-sentada 218 $(47,4 \%)$ e $217(47,2 \%)$, respectivamente. A maioria das mulheres respondeu que não foi preciso usar nenhuma intervenção para ajudar na saída do bebê 380 (82,6\%). A utilização da manobra de Kristeller no período expulsivo foi mencionada por 71 (15,5\%) delas. A prática da episiotomia foi mencionada por 127 (32,8\%) das puérperas (Tabela 2$)$.

Tabela 2 - Distribuição numérica e proporcional de práticas no parto normal, claramente prejudiciais ou ineficazes e que devem ser eliminadas, segundo OMS. Rio Branco, AC, Brasil, 2014

Práticas no parto normal, claramente prejudiciais ou ineficazes e que devem ser
eliminadas

Na análise, 54,2\% tiveram suas membranas amnióticas rompidas artificialmente, destas 30,7\% tinha dilatação uterina menor ou igual a cinco centímetros, no momento do rompimento das membranas. Quanto ao uso de anestesia durante o TP, a mais utilizada foi a local na região perineal (59,8\%) (Tabela $3)$. 
Tabela 3 - Distribuição numérica e proporcional segundo as recomendações da OMS, de práticas no parto normal frequentemente utilizadas de modo inapropriado. Rio Branco, AC, Brasil, 2014

\begin{tabular}{|c|c|c|c|}
\hline \multicolumn{2}{|c|}{ Práticas no parto normal frequentemente utilizadas de modo inapropriado } & \multirow{2}{*}{$\frac{\mathbf{N}}{249}$} & \multirow{2}{*}{$\frac{\%}{54,2}$} \\
\hline Ruptura das membranas amnióticas & Foi rompida artificialmente & & \\
\hline & Espontaneamente & 162 & 35,2 \\
\hline & Não, rompeu antes de chegar ao hospital & 37 & 8 \\
\hline & Não sabe & 12 & 2,6 \\
\hline \multirow{4}{*}{$\begin{array}{l}\text { Dilatação cervical, quando bolsa } \\
\text { rompeu }\end{array}$} & $\leq 5 \mathrm{~cm}$ & 111 & 30,7 \\
\hline & 6 a $7 \mathrm{~cm}$ & 75 & 20,7 \\
\hline & 8 a $9 \mathrm{~cm}$ & 137 & 37,8 \\
\hline & $10 \mathrm{~cm}$ & 39 & 10,8 \\
\hline \multirow[t]{4}{*}{ Anestesia durante o TP e P } & Sim, no períneo & 275 & 59,8 \\
\hline & Sim, nas costas & 5 & 1,1 \\
\hline & Não & 178 & 38,7 \\
\hline & Sim, geral & 2 & 0,4 \\
\hline \multirow[t]{3}{*}{ Revisão uterina após o $\mathrm{P}$} & Inspeção da vulva & 447 & 97,2 \\
\hline & Exploração manual & 5 & 1,1 \\
\hline & Não informado & 8 & 1,7 \\
\hline
\end{tabular}

As puérperas $433(94,1 \%)$ fizeram o contato pele a pele com recém-nascido logo após o parto e $392(85,2 \%)$ foram orientadas a amamentar, ainda no hospital, tendo uma média de dez minutos para colocar o bebê no peito pela primeira vez. Dos recém-natos 407 (88,5\%) permaneceram com suas mães durante todo o tempo de internação (Tabela 4).

Tabela 4 - Distribuição numérica e proporcional, com base nas recomendações da OMS, segundo as práticas na atenção ao recém-nascido. Rio Branco, AC, Brasil, 2014

\begin{tabular}{|c|c|c|c|}
\hline \multicolumn{2}{|l|}{ Práticas na atenção ao recém nascido } & \multirow{2}{*}{$\frac{\mathbf{N}}{433}$} & \multirow{2}{*}{$\frac{\%}{94,1}$} \\
\hline RN foi colocado contato pele a pele junto à puérpera & Sim & & \\
\hline & Sim, mas breve tempo & 19 & 4,1 \\
\hline & Não & 8 & 1,8 \\
\hline \multirow[t]{2}{*}{ No hospital, foi orientada a amamentar } & Sim & 392 & 85,2 \\
\hline & Não & 68 & 14,8 \\
\hline \multirow{2}{*}{$\begin{array}{l}\text { Quantos minutos demorou até a gestante dar o peito } \\
\text { pela primeira vez? }(\mathrm{N}=423)\end{array}$} & Mediana $\left(1^{\circ}\right.$ quartil $-3^{\circ}$ quartil $)$ & \multicolumn{2}{|c|}{$10(5-30)$} \\
\hline & Mínimo - Máximo & \multicolumn{2}{|c|}{$1-1320$} \\
\hline \multirow[t]{3}{*}{ Local em que o RN fica após o parto } & Com a puérpera & 407 & 88,5 \\
\hline & UCI/UTI ou berçário & 26 & 5,6 \\
\hline & $\begin{array}{l}\text { Parte do tempo com puérpera e } \\
\text { outra no berçário }\end{array}$ & 15 & 3,3 \\
\hline
\end{tabular}

\section{DISCUSSÃO}

O jejum durante o trabalho de parto é uma recomendação antiga e baseava-se na preocupação com o risco de aspiração do conteúdo gástrico, caso houvesse necessidade de uma anestesia geral. A restrição alimentar ainda é prescrita às mulheres no período que antecede o parto em algumas instituições de saúde (em Goiânia $62 \%$ das parturientes tiveram prescrição de dieta zero) ${ }^{(8)}$. Neste 
estudo, a liberdade de se alimentar durante o TP foi confirmada por $75 \%$ das mulheres, portanto, restrições alimentares não fazem parte da rotina da instituição.

A presença do acompanhante faz parte das condutas que devem ser estimuladas, essa prática está incorporada na rotina da instituição estudada: $87 \%$ tiveram acompanhante, atendendo à Lei $11.108 / 2005^{(9)}$, que obriga as instituições a permitir a presença junto à parturiente de um acompanhante durante todo o período de TP, parto e pós-parto imediato, devendo ser o indicado pela parturiente.

Vários estudos comprovaram que a presença do acompanhante proporciona benefícios ao binômio mãe-filho ${ }^{(10)}$. No entanto, a pesquisa nacional Nascer no Brasil mostrou que somente 18,8\% das mulheres experimentaram a presença de acompanhante de forma contínua enquanto estiveram na maternidade ${ }^{(11)}$.

O uso de métodos não farmacológicos para alívio da dor que são medidas que promovem conforto aumentando a tolerância à dor durante no processo do TP, permitindo que a mulher participe ativamente desse processo ${ }^{(2,12)}$. Dentre estes temos: movimentação a deambulação, uso da bola suíça, massagem de conforto, banho de aspersão e cavalinho.

A movimentação e a deambulação são práticas que devem ser estimuladas no $\operatorname{TP}^{(2)}$. Nesse estudo, a deambulação, apesar de ter sido amplamente estimulada 374 (81,3\%), obteve pouca adesão, somente $201(43,7 \%)$ puérperas aderiram à prática. Um estudo sobre os efeitos da deambulação no TP mostrou que esta proporciona benefícios tanto para a mãe quanto para o feto, pois auxilia na contração uterina, no fluxo sanguíneo útero placenta melhorando a oxigenação fetal, diminui a percepção da dor e o tempo do TP(13).

Este estudo mostra ainda que a distância percorrida durante a fase ativa também exerce grande influência no tempo de evolução do TP(13):

[...] a distância percorrida nas três primeiras horas da fase ativa do trabalho de parto esteve associada à redução no tempo desta fase. Esta redução foi de 22 minutos, 10 minutos e 6 minutos a cada 100 metros deambulado, na primeira, segunda e terceira hora, respectivamente, apontando para uma redução média de 2,04 horas, 1,67 hora e 1,34 hora, respectivamente às três primeiras horas da fase ativa do trabalho de parto [...]

Revisão sistemática publicada em 2013 sobre posição de parto destacou que o TP foi uma hora mais curto para as mulheres que se mantiveram na posição vertical, comparadas com as que adotaram a posição supina ou deitada de lado(13-14).

Movimentar-se durante o TP recebe forte recomendação pelo MS como medida não invasiva e não farmacológica para alívio da dor ${ }^{(2,7,13-14)}$. Os autores acreditam que podem reduzir e postergar a utilização de fármacos no controle da dor, proporcionar condições para a colaboração da parturiente, além disso permite participação do acompanhante ${ }^{(2)}$.

O uso da bola suíça vem recebendo forte recomendação por ajudar na evolução do $\operatorname{TP}^{(15)}$, no presente estudo, a bola foi adotada em 70\% (245) das mulheres entrevistadas. Ela atua direcionando a gestante para a posição vertical, facilitando a movimentação da pelve e trabalhando os músculos do assoalho pélvico ${ }^{(16)}$. Um ensaio clínico randomizado (ECR) em dois grupos de mulheres que reuniam as mesmas características obstétricas, tendo um usado bola suíça e o outro não, mostrou eficácia no alívio da dor para o grupo que usou a bola, mesmo não tendo influenciando no tempo de evolução do $\mathrm{TP}^{(15)}$. Outro estudo da mesma natureza realizado em Hong Kong e uma revisão sistemática com meta análise de três ECR corroboram com este achado ${ }^{(17-18)}$.

Quanto à massagem de conforto, nesse estudo 231 (64\%) parturientes relataram ter recebido massagem de conforto durante o TP. Em revisão sistemática ${ }^{(19)}$ quatro ECR referiam uso da massagem aplicado por parteira e acompanhante, mostrando-se mais efetiva quando utilizada no início da fase latente. Ela atua diminuindo os níveis de stress e ansiedade provocados pela dor, proporcionando também a participação ativa do acompanhante, melhorando a satisfação para ambos, corroborando com um estudo ${ }^{(19-20)}$.

Outra prática não farmacológica verificada foi o banho no chuveiro, relatado por 227 (63\%) participantes. Estudos vêm demonstrando que o banho de chuveiro em água quente é uma medida não farmacológica simples que promove relaxamento e conforto durante $\mathrm{O} \operatorname{TP}^{(12,21-22)}$. Além disso, quando aplicado nos 08 a $09 \mathrm{~cm}$ de dilatação uterina se mostrou efetivo no alívio da dor ${ }^{(22)}$. Assim, a 
combinação de dois ou mais métodos não farmacológicos mostrou-se ainda mais eficaz no alívio da dor e do desconforto durante o TP como caso da junção das terapia de banho e bola ${ }^{(16)}$.

O partograma é amplamente aceito como método útil para avaliação do progresso do TP. De acordo com a OMS, o uso do partograma permite acompanhar a evolução do TP, indicando a tomada de condutas apropriadas para a correção dos desvios e evitando intervenções desnecessárias ${ }^{(2)}$.

Um estudo realizado em 13 maternidades de Goiânia em 404 mulheres de parto normal, demonstrou a presença do partograma nos prontuários de apenas em $28,5 \%$ dos participantes, sendo que em $13 \%$ desses não havia qualquer anotação ${ }^{(8)}$. Outro estudo feito em uma maternidade de rede pública de São Paulo mostrou um número considerável de utilização incorreta desse instrumento dentro de um centro obstétrico $(77 \%)$, apesar de ele ter sido encontrado em quase $80 \%$ dos prontuários ${ }^{(23)}$.

Esse estudo identificou que 246 (53,5\%) prontuários tinham registro no partograma, destes somente $141(57,3 \%)$ foram parcialmente preenchidos, não sendo avaliada a qualidade do preenchimento. É importante destacar que este instrumento, apesar de não ser valorizado, é eficaz e necessário para avaliação da evolução do TP.

O uso de enema, tricotomia e administração de líquidos por via intravenosa são práticas consideradas claramente prejudiciais ou ineficazes e que devem ser eliminadas. Uma revisão sistemática sobre tricotomia pubiana no TP mostrou que não existem evidências que recomendem sua utilização de rotina $^{(7)}$.

Nesse estudo, não identificamos o uso do enema e da tricotomia em ambiente intra-hospitalar, no entanto, parece haver algo cultural entre as mulheres, pois a grande maioria delas realiza a raspagem dos pelos pubianos em seu domicílio (88\%) como parte da preparação para parto antes de buscar o hospital.

Quanto à infusão intravenosa de líquido e agentes uterotônicos, identificou-se que 223 (48,5\%) mulheres receberam infusão de líquidos intravenosos, e 139 (49,3\%) desconheciam o motivo da infusão. Destas, 87 (39\%) foram para administração de agentes uterotônicos, mesmo sendo uma prática considerada claramente ineficaz e que deve ser eliminada ${ }^{(2,7)}$. Apesar de ser uma prática prejudicial, continua sendo amplamente utilizada nas maternidades brasileiras: a pesquisa Nascer no Brasil demonstrou que $70 \%$ das mulheres fez uso de solução intravenosa durante o TP, sendo $40 \%$ com presença de agentes uterotônicos ${ }^{(11)}$.

A amniotomia, mesmo sendo uma prática não recomendada como rotina, vem sendo praticada como parte do manejo ativo do trabalho de parto por algumas escolas médicas ${ }^{(2,7)}$.

Uma revisão sistemática associou amniotomia a uma tendência ao risco da operação cesariana ${ }^{(24)}$. Neste estudo, a amniotomia foi realizada em 249 (54,2\%) das parturientes, sendo 162 (30,7\%) com cinco centímetros ou menos de dilatação. O uso da amniotomia é controverso e apresenta diversas realidades nas maternidades brasileiras, em um panorama nacional ela foi encontrada em $39 \%$ dos partos $^{(4)}$ já um estudo de Belo Horizonte destaca que sua taxa foi de $65 \%{ }^{(25)}$. Em estudo realizado no Rio de Janeiro em centro obstétrico onde os partos são atendidos exclusivamente por Enfermeiras Obstétricas, a taxa de amniotomia foi somente de 5,8\% ${ }^{(26)}$.

A posição da mulher durante o nascimento vem recebendo destaque conforme os avanços das pesquisas. Cada vez mais se tem provado que a posição litotômica prejudica tanto a parturiente como o feto durante o nascimento. A OMS e MS desencorajam o uso dessa posição ${ }^{(2,7)}$. No entanto, por muitas décadas, foi considerada a mais adequada para a realização de procedimentos hospitalares e adotada como clássica para o nascimento. As evidências científicas demonstraram seu malefício.

Contudo, muitas maternidades do Brasil ainda a adotam de forma rotineira não oferecendo opção à mulher de escolher a posição desejada para seu parto ${ }^{(2,7)}$. Pesquisa feita para analisar os resultados perineais de partos realizados nas posições vertical, semi sentada e lateral, conclui que partos na posição lateral apresentaram menor edema vulvar, episiotomia e laceração de lábios. Na posição vertical houve um maior risco de laceração de $2^{\circ} \operatorname{grau}^{(27)}$.

Um estudo realizado no Rio de Janeiro com 1.715 partos atendidos por enfermeiras obstétricas demonstrou $29,1 \%$ de episiotomia, neste as posições mais adotadas para o parto foram as verticais 
$(63,4 \%)$, sendo que $24,3 \%$ apresentou laceração de $1^{\circ}$ grau e $2,4 \%$ de $2^{\circ}$ grau, as lacerações dos partos na posição horizontal foram na ordem de $9,4 \%$ para as $1^{\circ}$ grau e somente $0,7 \%$ de $2^{\circ} \operatorname{grau}^{(28)}$.

Esta pesquisa evidenciou que a posição mais adotada para o parto foi deitada - 218 (47,4\%) e semi-sentada - 217 (47,2\%) no leito PPP (pré parto, parto e pós-parto), prevalecendo assim a posição horizontal. Uma revisão sistemática publicada pela biblioteca Cochrane em 2012 destaca que nas culturas tradicionais, as mulheres naturalmente têm seus partos em posições verticais, ajoelhadas, em pé ou de cócoras ${ }^{(14)}$.

Outra prática considerada claramente prejudicial, que deve ser eliminada, é a manobra de Kristeller, técnica que aplica uma pressão manual na parte superior do útero, utilizada em situações como sofrimento fetal, falta de progresso do parto e exaustão materna, a qual oferece riscos e por isso deve ser desencorajada ${ }^{(8)}$. Trata-se de uma conduta extremamente arriscada que causa dor e desconforto, violando o direito a mulher à sua integridade corporal ${ }^{(25)}$. Um estudo conduzido em uma maternidade na capital de Minas Gerais, identificou a realização dessa manobra em 9,3\% dos partos assistidos por enfermeiras obstétricas, sendo a taxa nacional de $36 \%{ }^{(25)}$. Neste estudo esta prática foi identificada em $71(15,5 \%)$ dos partos.

A ocorrência da episiotomia é um importante indicador de qualidade da atenção obstétrica. A evidência Cochrane mais recente sobre o uso seletivo de episiotomia versus uso rotineiro traz em seus achados que o uso seletivo está associado a menor risco de trauma perineal grave, menos perda sanguínea, apresentando pouco ou nenhum efeito sob o índice de Apgar ${ }^{(29)}$. Também traz que não houve diferença entre os dois grupos com relação à incontinência urinária de esforço, dispareunia e dor perineal após o parto, corroborando com as recomendações do MS que recomenda o uso restrito desta prática ${ }^{(7,29)}$.

O uso rotineiro da episiotomia está classificado de acordo com as evidências científicas como uma prática claramente prejudicial que deve ser eliminada ${ }^{(2,7)}$.

Neste estudo, a frequência de episiotomia foi de 127 (32,8\%), não existindo protocolo específico que recomendasse o uso seletivo ou rotineiro, ficando a critério do profissional a prática. Estudos realizados em duas capitais diferentes do país, com partos realizados prioritariamente por enfermeiras obstétricas, revelaram uma taxa de $8,4 \%$ e $29,1 \%{ }^{(25,28)}$. A pesquisa Nascer no Brasil de 2012 mostra uma taxa nacional de $56,1 \%$ dos partos ${ }^{(11)}$.

O contato pele a pele entre mãe e recém-nascido e a promoção do aleitamento materno na primeira hora de vida foram instituídas como uma das diretrizes para a organização da atenção integral e humanizada ao recém-nascido (RN) no SUS através de portaria ministerial ${ }^{(30)}$. O estudo Nascer no Brasil demonstra que o contato pele a pele ficou restrito a $26,6 \%$ dos RNs, somente $14,7 \%$ foi oferecida a mama ainda na sala de parto ${ }^{(11)}$.

No presente estudo, este cuidado recebe destaque, onde 433 (94,1\%) dos RNs permaneceram com as mães logo após o parto por mais de uma hora. Destas $392(85,2 \%)$ foram orientadas a amamentar ainda na sala de parto com uma mediana de 10 minutos para o início da amamentação.

Dos recém-nascidos participantes do estudo, 407 (88,5\%) permaneceram com suas mães durante todo o período de internação, os demais não ficaram com suas mães por estarem em condições de risco exigindo cuidados intensivos. Corroborando com os resultados deste estudo, dados revelaram que em uma maternidade da capital mineira nos partos vaginais o contato precoce entre o binômio foi garantido em mais de $77 \%$ dos partos, tendo mais de $90 \%$ dos recém-nascidos apresentado índices de Apgar maiores ou igual a oito ${ }^{(25)}$. Todavia, apesar deste e outros estudos evidenciar boas práticas de atenção ao recém nascido e ótimos desfechos perinatais, a pesquisa Nascer no Brasil demonstra que a prática do alojamento conjunto foi realizada em somente $64,2 \%$ dos nascimentos ${ }^{(11)}$.

Esta pesquisa apresenta a limitação relacionada à identificação do profissional responsável direto pela assistência ao parto, como os profissionais não utilizavam nenhum tipo de identificação que diferencie médicos de enfermeiros ou mesmo técnicos de enfermagem, não foi possível demonstrar qual categoria profissional cuidou da mulher durante o TP ou P. Recomenda-se, portanto, que os profissionais utilizem algum tipo de identificação ou instrumento que registre essa informação para que em estudos futuros e também a parturiente consiga definir e informar quem a atendeu, podendo 
assim verificar se existe diferença ou não na atuação profissional.

\section{- CONCLUSÃO}

Este estudo demonstra que a política de humanização implementada pelo MS e pela OMS no SUS em parte tem surtido efeito, pois encontramos boas práticas e medidas de conforto no trabalho de parto totalmente inseridas a rotina da instituição.

Todavia, demonstra que romper com um modelo tecnocrático e intervencionista não é tarefa fácil, intervenções inadequadas no processo fisiológico do TP e parto, como uso de solução com ocitócitos e indução com uterotônicos, posição horizontal no expulsivo, utilização da manobra de Kristeller e a episiotomia, ainda fazem parte das práticas obstétricas.

A supervalorização da prática diária e da experiência sobre as evidências científicas reforçam a importância do envolvimento das instituições que prestam assistência ao parto na (re)estruturação dos seus serviços, não só em estrutura física, mas principalmente na formação e capacitação de seus profissionais, para reformulação de antigos conceitos, devolvendo às mulheres o direito de serem protagonistas do nascimento de seus filhos.

\section{REFERÊNCIAS}

1. Nagahama EEI, Santiago SM. Childbirth practices and challenges for humanization of care in two public hospitals in Southern Brazil. Cad Saude Publica [Internet]. 2008 [acesso em 2017 maio 21]; 24(8). Disponível em: http://dx.doi.org/10.1590/S0102-311X2008000800014.

2. World Health Organization (WHO). Maternal and Newborn Health/Safe Motherhood Unit. Care in normal birth: a practical guide. [Internet]. Genebra: WHO; 1996 [acesso em 2016 jun 12]. Disponível em: https:// onlinelibrary.wiley.com/doi/abs/10.1111/j.1523-536X.1997.00121.pp.x.

3. Zanardo GLP, Uribe MC, Nadal AHR, Habigzang LF. Obstetrical violence in brazil: a narrative review. Psicol. Soc. [Internet]. 2017 [acesso em 2018 maio 15]; 29. Disponível em: http://dx.doi.org/10.1590/1807-0310/2017v29155043.

4. Leal MC, Pereira APE, Domingues RMSM, Theme Filha MM, Dias MAB, Nakamura-Pereira M, et al. Obstetric interventions during labor andchildbirth in Brazilian low-risk women. Cad Saude Publica. [Internet]. 2014 [acesso em 2018 maio 15]; 30(Suppl 1) Disponível em: http://dx.doi.org/10.1590/0102-311X00151513.

5. Dotto LMG. Saúde Reprodutiva de Primigestas: Análise de Fatores Relacionados ao Tipo de Parto [Pós Doutorado]. Ribeirão Preto (SP): Escola de Enfermagem de Ribeirão Preto, Universidade de São Paulo; 2011.

6. Ministério da Saúde (BR). Portaria n ${ }^{\circ}$ 1.459, de 24 de junho de 2011. Institui, no âmbito do Sistema Único de Saúde - SUS - a Rede Cegonha. Diário Oficial República Federativa do Brasil, 27 jun. 2011; Seção 1:109.

7. Ministério da Saúde. Parto, aborto e puerpério: assistência humanizada à mulher. [Internet]. Brasília; Ministério da Saúde; 2001 [acesso em 2017 ago 23]. Disponível em: http://bvsms.saude.gov.br/bvs/publicacoes/cd04_13.pdf.

8. Giglio MRP, França E, Lamounier JA. Evaluation of the quality of care for normal delivery. Rev Bras Ginecol Obstet. [Internet]. 2011 [acesso em 2016 jun 10]; 33(10). Disponível em: http://dx.doi.org/10.1590/S010072032011001000005.

9. Brasil. Lei n. 11.108, de 7 de abril de 2005. Altera a Lei no 8.080, de 19 de setembro de 1990, para garantir às parturientes o direito à presença de acompanhante durante o trabalho de parto, parto e pós-parto imediato, no âmbito do Sistema Único de Saúde - SUS. Oficial da República Federativa do Brasil, Brasília, 07 abr. 2005.

10. Bruggemann O, Parpinelli MA. O apoio a mulher no nascimento por acompanhante de sua escolha: abordagem quantitativa e qualitativa [Tese de Doutorado]. Campinas (SP): Universidade Estadual de Campinas; 2005.

11. Diniz CSG, d'Orsi E, Domingues RMSM, Torres JA, Dias MAB, Schneck CA, et al. Implementation of the presence of companions during hospital admission for childbirth: data from the Birth in Brazil national 
survey. Cad Saude Publica [Internet]. 2014 [acesso em 2018 maio 17]; 30(Suppl 1). Disponível em: http://dx.doi. org/10.1590/0102-311X00127013.

12. Coelho KC, Rocha IMS, Lima ALS. Non-pharmacological methods for pain relief during labor. Rev Recien. [Internet]. 2018 [acesso em 2018 maio 15]; 8(22). Disponível em: http://dx.doi.org/10.24276/ rrecien2358-3088.2018.8.22.14-21.

13. Mamede FV, de Almeida AM, Nakano AMS, Gomes FA, Panobianco MS. The ambulation effect in the active stage duration of the labor. Esc. Anna Nery. [Internet]. 2007 [acesso em 2016 jun 10]; 11(3). Disponível em: http:// dx.doi.org/10.1590/S1414-81452007000300011.

14. Lawrence A, Lewis L, Hofmeyr GJ, Dowswell T, Styles C. Maternal positions and mobility during first stage labour. Cochrane Database of Systematic Reviews. [Internet]. 2013 [acesso em 2018 maio 15]; (8). Disponível em:https://doi.org//10.1002/14651858.CD003934.pub3.

15. Gallo RBS, Santana LS, Marcolin AC, Quintana SM. Swiss ball to relieve pain of primiparous in active labor. Rev. Dor. [Internet]. 2014 [acesso em 2018 maio 15]; 15(4). Disponível:http://dx.doi.org/10.5935/1806-0013.20140054.

16. Barbieri M, Henrique AJ, Chors FM, Maia NL, Gabrielloni MC. Warm shower aspersion, perineal exercises with Swiss ball and pain in labor. Acta Paul. Enferm. [Internet]. 2013 [acesso em 2016 jun 10]; 26(5). Disponível em: http://dx.doi.org/10.1590/S0103-21002013000500012.

17. Makvandi S, Latifnejad Roudsari R, Sadeghi R, Karimi L. Effect of birth ball on labor pain relief: A systematic review and meta-analysis. J Obstet Gynaecol Res. [Internet]. 2015 [acesso em 21 mai 2017]; 41(11). Disponível em: https://www.ncbi.nlm.nih.gov/pubmed/26419499.

18. Leung RWC, Li JFP, Leung MKM, Fung BKY, Fung LCW, Tai SM, et al. Efficacy of birth ball exercises on labour pain management. Hong Kong Med J Xianggang Yi Xue Za Zhi. Med J Hong Kong. [internet]. 2013 [acesso em 2018 maio 15]; 19(5) Disponível em: http://dx.doi.org/10.12809/hkmj133921.

19. Gayeski ME, Brüggemann OM. Non-pharmacological approach to pain relief during labor as hard-light care technology: a systematic review. Texto contexto - enferm. [Internet]. 2010 [acesso em 2018 maio 15]; 19(4). Disponível em: http://dx.doi.org/10.1590/S0104-07072010000400022.

20. Chang M-Y, Wang S-Y, Chen C-H. Effects of massage on pain and anxiety during labour: a randomized controlled trial in Taiwan. J Adv Nurs. [Internet]. 2002 [acesso em 2018 maio 16]; 38(1) Disponível em: https://doi. org/10.1046/j.1365-2648.2002.02147.x.

21. Mazoni SR, de Faria DGS, Manfredo VA. Hidroterapia durante o trabalho de parto: relato de uma prática segura. Arq Ciênc Saúde. [Internet]. 2009 [acesso em 2018 maio 15]; 16(1). Disponível em: http://repositorio-racs. famerp.br/racs_ol/vol-16-1/ID_305.pdf.

22. Silva EF, Strapasson MR, Fischer ACS. Non-pharmacological methods of pain relief during labor and delivery. Rev. enferm. UFSM. [Internet]. 2011 [acesso em 2018 maio 15]; 1(2). Disponível em: http://dx.doi. org/10.5902/217976922526.

23. Aguiar CA. Práticas obstétricas e a questão das cesarianas intraparto na rede pública de saúde São Paulo [Dissertação de Mestrado]. São Paulo (SP): Faculdade de Saúde Pública da Universidade de São Paulo; 2012.

24. Porto AMF, Amorim MMR, Souza ASR. Assistência ao primeiro período do trabalho de parto baseada em evidências: [revisão]. Femina. [Internet]. 2010 [acesso em 2017 jun 14]; 38(10). Disponível em: http://bases.bireme. br/cgi-bin/wxislind.exe/iah/online/?IsisScript=iah/iah.xis\&src=google\&base=LILACS\&lang=p\&nextAction=Ink\& exprSearch=574503\&indexSearch=ID.

25. Sousa AMM, Souza KV, Rezende EM, Martins EF, Campos D, Lansky S. Practices in childbirth care in maternity with inclusion of obstetric nurses in Belo Horizonte, Minas Gerais. Esc Anna Nery. [Internet]. 2016 [acesso em 2016 jun 13]; 20(2). Disponível em: http://www.gnresearch.org/doi/10.5935/1414-8145.20160044.

26. Rocha CR, Fonseca LC. The assistence of parturient women by obstetric nurses: in search for the respect of nature. Dialnet. [Internet]. 2010 [acesso em 2016 jun 10]; 2(2). Disponível em:http://dx.doi.org/10.9789/21755361.2010.v2i2.\%p. 
27. Schirmer J, Fustinoni SM, Basile AL de O. Perineal outcomes on the left lateral versus vertical semi-sitting birth positions: a randomized study. Acta Paul. Enferm. [Internet]. 2011 [acesso em 2016 jun 10]; 24(6). Disponível em: http://dx.doi.org/10.1590/S0103-21002011000600002.

28. Mouta RJO, Pilotto DT dos S, Vargens OM da C, Progianti JM. Relação entre a posição adotada pela mulher no parto, integridade perineal e vitalidade do recém-nascido. Rev. enferm. UER]. [Internet]. 2008 [acesso em 2018 jun 16]; 16(4). Disponível em: http://www.facenf.uerj.br/v16n4/v16n4a03.pdf.

29. Jiang H, Qian X, Carroli G, Garner P. Selective versus routine use of episiotomy for vaginal birth. Cochrane Database Syst Rev. [Internet]. 2017 [acesso em 2018 maio 18]; (2) Disponível em: http://dx.doi.org/10.1002/14651858. CD000081.pub3.

30. Ministério da Saúde (BR). Portaria n. 371, de 7 de maio de 2014. Institui diretrizes para a organização da atenção integral e humanizada ao recém nascido no Sistema Único de Saúde. Diário Oficial da União, 7 maio 2014. 